\title{
Statistics of linear polymer chains in the self-avoiding walks model
}

\author{
Yu.G.Medvedevskikh \\ The Department of the L.V. Pisarzhevsky Institute of Physical Chemistry \\ of the National Academy of Sciences of Ukraine, \\ 3a Naukova Str., 79053 Lviv, Ukraine
}

Received December 20, 2000

\begin{abstract}
A strict statistics of self avoiding random walks in $d$-dimensional discrete (lattice) and continuous space is proposed. Asymptotic analytical expressions for the distribution and distribution density of corresponding random values characterizing a conformational state of polymer chain have been obtained and their quantitative estimation has been given. It is shown that conformation of polymer chain possesses a structure of spherical or, more commonly, of elliptical shell diffusely blurred both outside and inside the polymer coil, which nucleus is statistically void and has a radius of about half of Flory radius. Statistics of self-avoiding walks describes completely an effect of excluded volume and meets the terms of Flory method in Pietronero's concepti.
\end{abstract}

Key words: Flory method, statistics, self-intersection, polymer chain, random walks, lattice, fatigue function, conformation

PACS: $05.40 . F b$

\section{Flory method and Pietronero's concepti}

One of the peculiarities of long flexible polymer chains is that their thermodynamic and dynamic properties in solution are to a great extent determined not by the nature of links but by their quantity, i.e. by conformation of macromolecules. The basis of its description is a model of free-joined Kuhn's chain and statistics of random walks (RW) of its growing end [1]. In terms of those statistics, the distance distribution between the chain ends is described by Gaussian function having two features: distribution mode corresponds to zero distance between chain ends, and root-mean-square radius $R_{0}$ of polymer coil and chain length $N$ correlate as $R_{0} \sim N^{\nu}$, where $\nu=1 / 2$. Experimental data show the values close to $3 / 5$.

Taking into account the effects of short range ordering, e.g. of fixed valent angles and hinderance of internal rotation $[2,3]$, do not change the shape of distribution function and the correlation $R_{0} \sim N^{1 / 2}$ but increase $R_{0}$ that can be interpreted in terms of the increase of a statistical length of equivalent Kuhn's chain segment [4]. 
It was several times noticed $[2,3]$ that soft polymer chain configuration differs in one important relation from RW trajectory: it must not intersect. This limitation known as long range ordering effect or excluded volume effect demands new statistics, i.e., statistics of self-avoiding walks (SAW). The attempts made (see [2]) have not permitted to solve this problem completely.

That is why the authors of references $[5,6]$ find the success of Flory method (after de Gennes [7]) to be especially unexpected. In this method, the free energy $F$ of polymer chain conformation in $k T$ units is presented by a sum

$$
F=v N^{2} / R^{d}+d R^{2} / 2 a^{2} N
$$

where $v=a^{d}(1-2 \chi)$ is an excluded or an effective volume of monomer link, $a$ is its statistical length, $\chi$ is a Flory-Huggins parameter, $R$ is a distance between chain ends and $d$ is the Euclidean space dimension.

The augend in (1) represents a pairwise repulsion energy of chain links in the selfconsistent field approximation, and the addend represents the polymer chain elastic energy determined via entropy $(F=-T S)$ of its conformation in RW statistics.

Minimization of $F$ by $R$ permits to find the equilibrium or the most probable radius $R_{\mathrm{f}}$ of Flory coil:

$$
\begin{aligned}
R_{\mathrm{f}} & =a_{\mathrm{f}} N^{\nu} \\
\nu & =\frac{3}{(d+2)}
\end{aligned}
$$

where $a_{\mathrm{f}}=a(1-2 \chi)^{1 /(d+2)}$.

Formula (3) appears to be universal: it is in good accordance with the results of physical experiment and computer simulation of SAWs on a lattice [5-8].

Flory conception put in expression (1) cannot give a strict explanation for those results. That is why, Pietronero [5] has proposed another concepti of Flory method, in accordance with which, both components of expression (1) possess an entropic nature but the conformation entropy is determined by SAW statistics.

Pietronero's concepti makes explainable a good correlation of Flory method formula (3) with the results of SAW computer simulation on the lattice and gives a heuristic landmark for the construction of strict SAW statistics.

\section{Statistics of SAWs on a $d$-dimensional lattice}

Let us incorporate the numbers $n_{i}$ of chain end steps along the $i$ direction of $d$-dimensional lattice with cell size equal to statistical length of chain link. Besides:

$$
\sum_{i} n_{i}=N, \quad i=1, d
$$

The quantity of realization variants of random steps along the $i$ direction is equal to $n_{i} ! / n_{i}^{+} ! n_{i}^{-}$!, where the numbers of steps in positive $n_{i}^{+}$and negative $n_{i}^{-}$directions of wandering are correlated by $n_{i}^{+}+n_{i}^{-}=n_{i}$. Considering that the probability of 
wandering positive or negative direction choice is assumed to be the same and equal to $1 / 2$, the probability $\omega\left(n_{i}\right)$ that at given $n_{i}, n_{i}^{+}$positive and $n_{i}^{-}$negative steps will be made is determined by Bernoulli distribution:

$$
\omega\left(n_{i}\right)=\left(\frac{1}{2}\right)^{n_{i}} n_{i} ! / n_{i}^{+} ! n_{i}^{-} !
$$

Then, incorporating the quantity of effective steps $s_{i}=n_{i}^{+}-n_{i}^{-}$along $i$ direction of wandering, we obtain $n_{i}^{+}=\left(n_{i}+s_{i}\right) / 2, n_{i}^{-}=\left(n_{i}-s_{i}\right) / 2$. Then (5) can look like the following:

$$
\omega\left(n_{i}\right)=\left(\frac{1}{2}\right)^{n_{i}} n_{i} ! /\left(\left(n_{i}+s_{i}\right) / 2\right) !\left(\left(n_{i}-s_{i}\right) / 2\right) !
$$

For $d$-dimensional wandering we have

$$
\omega(n)=\left(\frac{1}{2}\right)^{N} \prod_{i} n_{i} ! /\left(\left(n_{i}+s_{i}\right) / 2\right) !\left(\left(n_{i}-s_{i}\right) / 2\right) ! .
$$

One can see that change of sign at $s_{i}$ does not change the value $\omega(n)$. That is why $\omega(n)$ represents the probability that the trajectory of random wandering after $n_{i}$ steps along $i$ directions will be finished in one of $2^{d}$ cells $M_{p}(s)$, which coordinates are determined by vectors $s=\left(s_{i}\right), i=1, d$ having a distinction in signs of their components $s_{i}$ only. These cells or states of chain end are equiprobable.

The condition of self-avoidance of a RW trajectory on $d$-dimensional lattice demands the step not to fall twice in the same cell. From the point of view of chain link distribution over cells it means that every cell cannot contain more than one chain link. Chain links are inseparable. They cannot be torn off one from another and placed to cells in random order. Consequently, the numbering of chain links corresponding to wandering steps is their significant distinction. That is why, the quantity of different variants of $N$ distinctive chain links placement in $Z$ identical cells under the condition that one cell cannot contain more than one chain link is equal to $Z ! /(Z-N)$ ! .

Considering the identity of cells, a priori probability that the given cell will be filled is equal to $1 / Z$, and that it will not be filled is $(1-1 / Z)$. Respectively, the probability $\omega(z)$ that $N$ given cells will be filled and $Z-N$ cells will be empty, considering both the above mentioned condition of placement of $N$ distinctive links in $Z$ identical cells and the quantity of its realization variants, will be determined by the following expression

$$
\omega(z)=Z !(1 / Z)^{N}(1-1 / Z)^{Z-N} /(Z-N) ! .
$$

Probability $\omega(s)$ of simultaneous event, meaning that the RW trajectory is also the SAW trajectory and, at given $Z, N$ and $n_{i}$, it will fall with its last step into one of $2^{d}$ equiprobable cells $M_{p}(s)$, will be equal to

$$
\omega(s)=\omega(z) \omega(n) .
$$


Let us find the asymptotic (9), assuming $Z \gg 1, N \gg 1, n_{i} \gg 1$ under the condition $s_{i} \ll n_{i}, N \ll Z$. Using the approximated Stirling formula $\ln x$ ! $\approx x \ln x-$ $x+\ln (2 \pi)^{1 / 2}$ for all $x \gg 1$ and expansion $\ln (1-1 / Z) \approx-1 / Z, \ln (1-N / Z) \approx-N / Z$, $\ln \left(1 \pm s_{i} / n_{i}\right) \approx \pm s_{i} / n_{i}-\left(s_{i} / n_{i}\right)^{2} / 2$, and assuming also $N(N-1) \approx N^{2}$, we will obtain

$$
\omega(s) \approx \beta \exp \left\{-N^{2} / Z-\frac{1}{2} \sum_{i} s_{i}^{2} / n_{i}\right\}
$$

where $\beta^{-1}=e(2 \pi)^{d / 2}$.

With the growth of the quantity $s_{i}$ of effective steps the chain end moves away from its origin increasing the conformational volume where those SAW trajectories are localized at that end in one of $2^{d}$ equiprobable cells $M_{p}(s)$. That is why the cells quantity $Z$, allowed for a SAW trajectory, is not a fixed parameter of distribution (10), but it is a function of vector $s=\left(s_{i}\right): Z=Z(s)$. This function choice can be made based on different geometrical estimations - see figure 1 . For instance, if one considers that conformational volume of SAW trajectories is localized in $d$ dimensional rectangle with vertexes $M_{p}(s)$, then $Z=2^{d} \prod_{i}\left|s_{i}\right|$. If one assumes that conformational volume is localized in the sphere with radius of $R_{s}=\left(\sum_{i} s_{i}^{2}\right)^{1 / 2}$ then the expression $Z=\left(\sum_{i} s_{i}^{2}\right)^{d / 2}$ can be used for a definition. At least, if one assumes that fiducial cells $M_{p}(s)$ belong to an ellipsoid surface, which semi-axes in accordance to canonical equation $\sum_{i} s_{i}^{2} / b_{i}^{2}=1$ must be equal to $b_{i}=d^{d / 2}\left|s_{i}\right|$, it can be laid: $Z=d^{d / 2} \prod_{i}\left|s_{i}\right|$. The analysis has shown that this definition is the most suitable (it will be confirmed below), that is why we assume

$$
Z=d^{d / 2} \prod_{i}\left|s_{i}\right| .
$$

Putting together (10) and (11), we have

$$
\omega(s)=\beta \exp \left\{-N^{2} / d^{d / 2} \prod_{i}\left|s_{i}\right|-\frac{1}{2} \sum_{i} s_{i}^{2} / n_{i}\right\}, \quad 1 \leqslant s_{i} \leqslant n_{i} .
$$

Function $\omega(s)$ determines the probability of a complicated event meaning that RW trajectory is also a SAW trajectory at the same time and with it last step it falls into one of $2^{d}$ equiprobable cells $M_{p}(s)$ or one can say that it realizes the state $M_{p}(s)$. This implies that it is numerically equal to the part of those SAW trajectories from the whole quantity of RW trajectories $(2 d)^{N}$, which realize the state $M_{p}(s)$. Quantity $L(s)$ of such trajectories define the thermodynamical probability of a realization of the state $M_{p}(s)$

$$
L(s)=(2 d)^{N} \omega(s) .
$$

Summing $L(s)$ over the whole set $\Omega$ of chain end states, we find the total quantity $L$ of SAW trajectories

$$
L=(2 d)^{N} \sum_{\Omega} \omega(s) .
$$

Designating

$$
c(s)=\sum_{\Omega} \exp \left\{-N^{2} / d^{d / 2} \prod_{i}\left|s_{i}\right|-\frac{1}{2} \sum_{i} s_{i}^{2} / n_{i}\right\}
$$




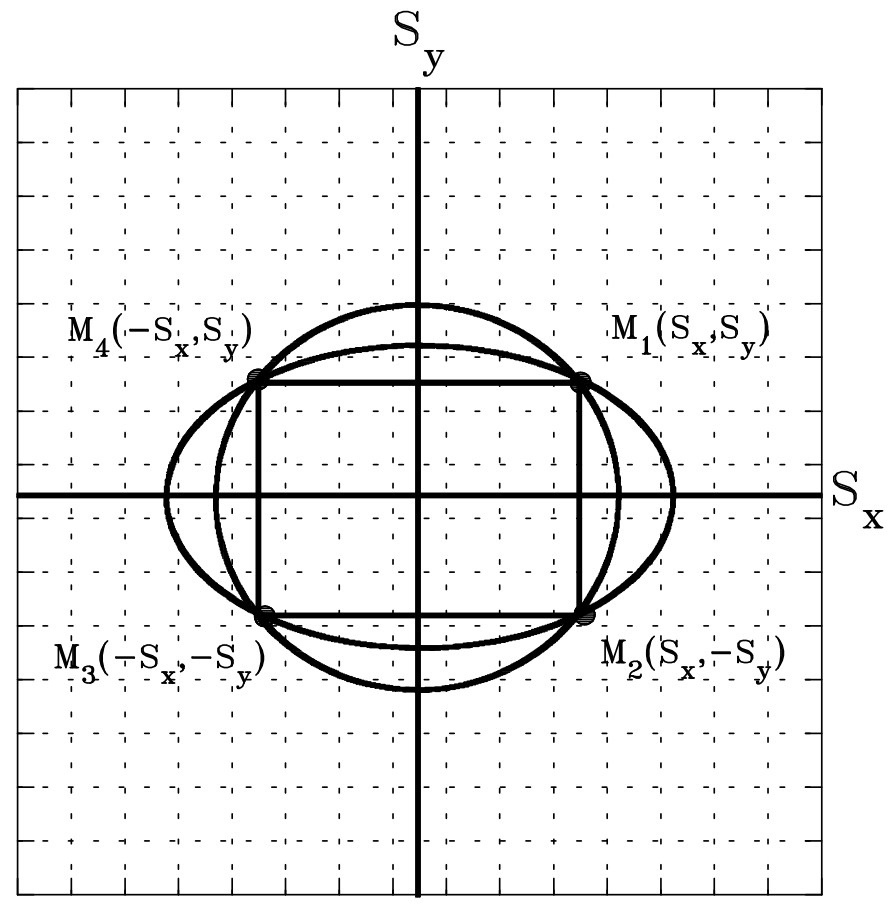

Figure 1. Geometrical variants of determination of RWEI trajectories conformational volume at given coordinates of equiprobable states $M_{p}(S)$ of chain end for a two-dimensional lattice.

the function

$$
w(s)=1 / c(s) \exp \left\{-N^{2} / d^{d / 2} \prod_{i}\left|s_{i}\right|-\frac{1}{2} \sum_{i} s_{i}^{2} / n_{i}\right\}
$$

is normalized to unity and define the mathematical probability of chain end distribution over the states $M_{p}(s)$ of $d$-dimensional lattice. It equals to the relation of quantity $L(s)$ of those SAW trajectories, which realize the state $M_{p}(s)$, to the total quantity of SAW trajectories $L: w(s)=L(s) / L$.

In turn, the ratio $L /(2 d)^{N}$ equals to the part of total number of SAW trajectories from the total number of RW trajectories and is a function $g(N)$ of SAW trajectories fatigue, in accordance to terminology [5,6], and quantitatively, it can be determined via the normalizing constant of distribution (16):

$$
g(N)=L /(2 d)^{N}=\beta c(s) .
$$

\section{Statistics of SAWs in continuous space}

Expressions (12)-(17) represent the SAW statistics at $d$-dimensional lattice. Let us work with a metric space, incorporating the variable of shifting the $x_{i}$-semi-axis of conformational ellipsoid, with the states $M_{p}(s)$ belonging to its surface

$$
x_{i}=a\left|s_{i}\right| d^{1 / 2}
$$


and parameter $\sigma_{i}$ is the standard deviation of the Gaussian part of the distribution

$$
\sigma_{i}^{2}=a^{2} n_{i} d
$$

In accordance with (4), the coupling is laid on the value $\sigma_{i}$

$$
\sum_{i} \sigma_{i}^{2}=a^{2} N d
$$

Since $s_{i}^{2} / n_{i}=x_{i}^{2} / \sigma_{i}^{2}, d^{d / 2} \prod_{i}\left|s_{i}\right|=\prod_{i} x_{i} / a^{d}$, the distribution (12) can be rewritten as follows

$$
w(x)=1 / c(x) \exp \left\{-a^{d} N^{2} / \prod_{i} x_{i}-\frac{1}{2} \sum_{i} x_{i}^{2} / \sigma_{i}^{2}\right\}, \quad a \leqslant x_{i} \leqslant a n_{i} .
$$

If $x_{i \min }=a \ll x_{i \max }=a n_{i}$, the distribution (21) can be considered as a continuous one with a normalizing constant

$$
c(x)=\int_{x_{\min }}^{x_{\max }} \exp \left\{-a^{d} N^{2} / \prod_{i} x_{i}-\frac{1}{2} \sum_{i} x_{i}^{2} / \sigma_{i}^{2}\right\} \mathrm{d} x, \quad a \leqslant x_{i} \leqslant a n_{i},
$$

where $\mathrm{d} x=\prod_{i} \mathrm{~d} x_{i}$, and the integral is $d$-aliquot. In this case: $c(x)=a^{d} d^{d / 2} c(s)$.

Substitution of (18) induces an essential distinction between $w(x)$ and $w(s): w(x)$ determines the probability $w(x) \mathrm{d} x$ that the SAW trajectory at given parameters $N$ and $\sigma_{i}$ will be ended in elementary volume $\mathrm{d} x$, laying on an ellipsoid surface with semi-axes $x_{i}, i=1, d$. In the other case, all the surface of ellipsoid is a geometrical place of points or chain end states with the same corresponding distribution density $w(x)$.

Maximum of $w(x)$ at given $N$ and $\sigma_{i}$ corresponds to the most probable or equilibrium state of a polymer chain. Semi-axes $x_{i}^{0}$ of equilibrium conformational ellipsoid can be found from the condition

$$
\partial \ln w(x) / \partial x_{i}=a^{d} N^{2} / x_{i} \prod_{i} x_{i}-x_{i} / \sigma_{i}^{2}=0 \quad \text { when } \quad x_{i}=x_{i}^{0} .
$$

Solving the system of algebraic equations (23), we obtain

$$
x_{i}^{0}=\sigma_{i}\left(a^{d} N^{2} / \prod_{i} \sigma_{i}\right)^{1 /(d+2)} .
$$

Let us continue to consider the situation when all the directions of chain steps are equiprobable, i.e.

$$
\begin{gathered}
n_{i}=N / d, \\
\sigma_{i}^{2}=\sigma_{0}^{2}=a^{2} N .
\end{gathered}
$$

Substitution of (26) into (24) makes the semi-axes of equilibrium ellipsoid equal, and equal to the radius of Flory coil

$$
x_{i}^{0}=R_{\mathrm{f}}=a N^{\nu},
$$

where $\nu$ is defined by (3). 


\section{Some properties of distribution in SAW statistics}

To avoid the utilization of parameter $a$, i.e., the length of chain link in calculations, we will use a dimensionless variable

$$
\lambda_{i}=x_{i} / R_{\mathrm{f}}
$$

Besides, let us consider only the distribution corresponding to the condition (25) of RW equiprobability over the axes of $d$-dimensional space. In this case the expressions (21) and (22) can be rewritten as follows

$$
\begin{aligned}
w(\lambda)= & 1 / c(\lambda) \exp \left\{-\left(R_{\mathrm{f}} / \sigma_{0}\right)^{2}\left(1 / \prod_{i} \lambda_{i}+\frac{1}{2} \sum_{i} \lambda_{i}^{2}\right)\right\}, \\
& c(\lambda)=I_{0}, \\
& I_{0}=\int_{\lambda_{\min }}^{\lambda_{\max }} \exp \left\{-\left(R_{\mathrm{f}} / \sigma_{0}\right)^{2}\left(1 / \prod_{i} \lambda_{i}+\frac{1}{2} \sum_{i} \lambda_{i}^{2}\right)\right\} \mathrm{d} \lambda,
\end{aligned}
$$

where $\mathrm{d} \lambda=\prod_{i} \mathrm{~d} \lambda_{i}, \lambda_{\min }=a d^{1 / 2} / R_{\mathrm{f}}, \lambda_{\max }=a N / R_{\mathrm{f}} d^{1 / 2}$. Normalizing constants of distributions (21) and (29) are correlated with $c(\lambda)=c(x) / R_{\mathrm{f}}^{d}$, that is why we have a coupling

$$
c(s)=\left(R_{\mathrm{f}} / a\right)^{d} c(\lambda) / d^{d / 2}=c(x) / a^{d} d^{d / 2} .
$$

In accordance to (26) and (27)

$$
\left(R_{\mathrm{f}} / \sigma_{0}\right)^{2}=N^{(4-d) /(d+2)} .
$$

Quantitative estimation of $I_{0}$ (and of the following ones) is performed using the Romberg's algorithm from Mathcad software with accuracy parameter of Tol = $10^{-4}$. Values $I_{0}$ are listed in the table.

Values $\lambda_{i}^{0}=1, i=1, d$, relating to maximal distribution density, correspond to the equilibrium state of polymer coil

$$
w\left(\lambda^{0}\right)=1 / c(\lambda) \exp \left\{-\left(R_{\mathrm{f}} / \sigma_{0}\right)^{2}(d+2) / 2\right\} .
$$

Distribution density decreases at any deviation of values $\lambda_{i}$ at $\lambda^{0}=1$ : it this case it tends to zero not only at $\lambda_{i} \rightarrow \lambda_{\max }$, but also at $\lambda_{i} \rightarrow \lambda_{\min }$. Consequently, the probability of chain end placement close to its origin is negligibly small. The latter makes a radical distinction between SAW statistics and gaussian RW statistics.

Figure 2 represents the illustrations for several variants of function $w(\lambda)$ behaviour for a three-dimensional space $d=3$ at $\left(R_{\mathrm{f}} / \sigma_{0}\right)^{2}=N^{1 / 5}$, equal to 3 and 4 , and changing of one variable $\lambda_{z}$ at fixed values of $\lambda_{x}=\lambda_{y}=1, \lambda_{x}=\lambda_{y}=0.8$ and $\lambda_{x}=\lambda_{y}=1.25$. The two latter variants clearly show what will happen at the deformation of polymer coil: at compression along $x$ and $y$ axes, polymer chain stretches along $z$ axis transforming to oblong ellipsoid of revolution; on the contrary, at compression along $z$ axis, polymer chain stretches along $x$ and $y$ axes transforming to oblate ellipsoid of revolution. 


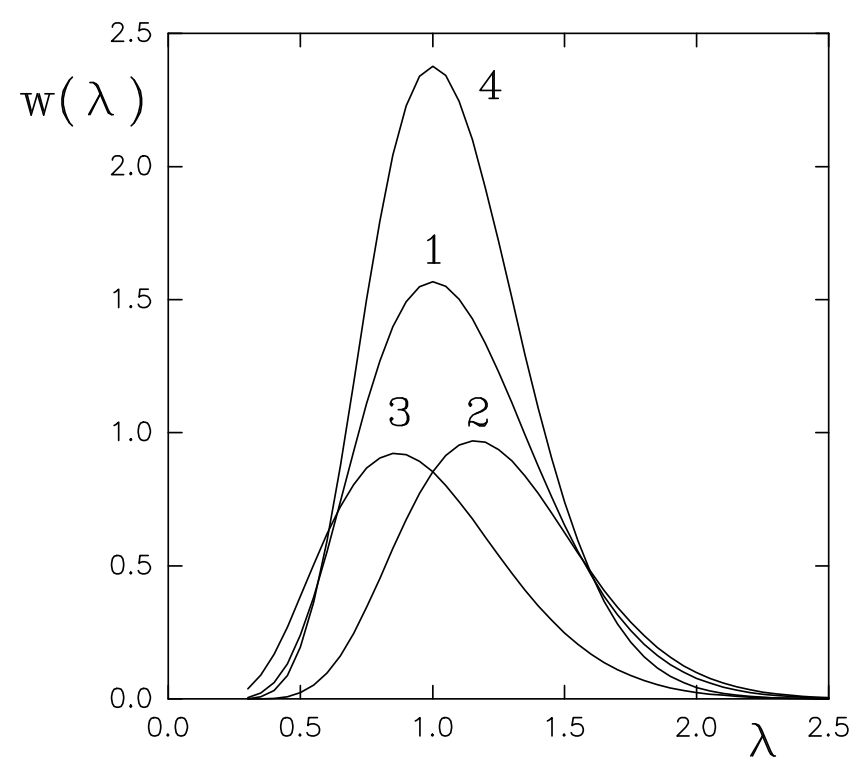

Figure 2. Behaviour of distribution density $w(\lambda)$ at the variation of $\lambda=\lambda_{z}$ and fixed values of $\lambda_{x}$ and $\lambda_{y}$ for $d=3 . N^{1 / 5}=3(1,2,3), N^{1 / 5}=4(4), \lambda_{x}=\lambda_{y}=$ $1(1,4), \lambda_{x}=\lambda_{y}=0.8(2), \lambda_{x}=\lambda_{y}=1.25(3)$.

Starting moments $M\left(\lambda^{k}\right)(k=1,2)$ of distribution (29) are numerically calculated (as it was mentioned before) using the correlation

$$
M\left(\lambda^{k}\right)=\int_{\lambda_{\min }}^{\lambda_{\max }} \lambda_{i}^{k} w(\lambda) \mathrm{d} \lambda
$$

Values $M\left(\lambda^{k}\right)$ for Flory coil do not depend on the index $i$. Root-mean-square value $\lambda_{0}=\left(M\left(\lambda^{2}\right)\right)^{1 / 2}$, dispersion $D(\lambda)=M\left(\lambda^{2}\right)-(M(\lambda))^{2}$ and standard deviation $\sigma(\lambda)=(D(\lambda))^{1 / 2}$ of distribution (29) are calculated via the values $M(\lambda)$ and $M\left(\lambda^{2}\right)$. A part of the data calculated is presented in the table.

Table 1. Some characteristics of distribution density $\mathrm{w}(\lambda)$

\begin{tabular}{|c|c|c|c|c|c|c|c|}
\hline$N^{1 / 5}$ & $I_{0}$ & $I_{0}(L)$ & $M(\lambda)$ & $M\left(\lambda^{2}\right)$ & $\lambda_{0}$ & $D(\lambda)$ & $\sigma(\lambda)$ \\
\hline 2 & $7.72 \cdot 10^{-3}$ & $8.39 \cdot 10^{-3}$ & 1.139 & 1.487 & 1.219 & 0.191 & 1.437 \\
\hline 4.0 & $1.91 \cdot 10^{-5}$ & $2.00 \cdot 10^{-5}$ & 1.073 & 1.246 & 1.116 & 0.094 & 0.307 \\
\hline 6.0 & $7.11 \cdot 10^{-8}$ & $7.33 \cdot 10^{-8}$ & 1.048 & 1.163 & 1.078 & 0.065 & 0.255 \\
\hline 8.0 & $3.13 \cdot 10^{-10}$ & $3.21 \cdot 10^{-10}$ & 1.035 & 1.125 & 1.060 & 0.053 & 0.230 \\
\hline 10.0 & $1.52 \cdot 10^{-12}$ & $1.55 \cdot 10^{-12}$ & 1.026 & 1.099 & 1.048 & 0.045 & 0.217 \\
\hline 12.0 & $7.79 \cdot 10^{-15}$ & $7.9 \cdot 10^{-15}$ & 1.024 & 1.083 & 1.041 & 0.034 & 0.185 \\
\hline
\end{tabular}




\section{The fatigue function}

The fatigue function $g(N)$ is defined by (17) and expresses the part of the total quantity of SAW trajectories from the total quantity of RW trajectories. The latter quantity $(2 d)^{N}$ is strictly defined for the lattice only, i.e. for the discrete space of wanderings. The total quantity of RW trajectories for continuous space is unknown, however the fatigue function can still be defined by (17) via normalizing constant $c(x)$. Considering coupling (32), we can express the fatigue function via normalizing constant $c(\lambda)$ :

$$
g(N)=\beta\left(R_{\mathrm{f}} / a\right)^{d} d^{-d / 2} c(\lambda) .
$$

One can use the quantitative values $c(\lambda)=I_{0}$ presented in the table for calculations. However, in case of $d<4$ space, one can give an approximated analytical expression $I_{0}$, using the Laplace's method [10]. Then, at $d=3$, the fatigue function can be written as follows

$$
g(N) \approx g_{0} N^{3 / 2} \exp \left\{-5 / 2 N^{1 / 5}\right\}
$$

where $g_{0}=\left(e 2 \sqrt{ } 53^{3 / 2}\right)^{-1}$. Function (37) possesses a maximum at $N^{1 / 5}=3$. Its graphical representation is given in figure 3 .

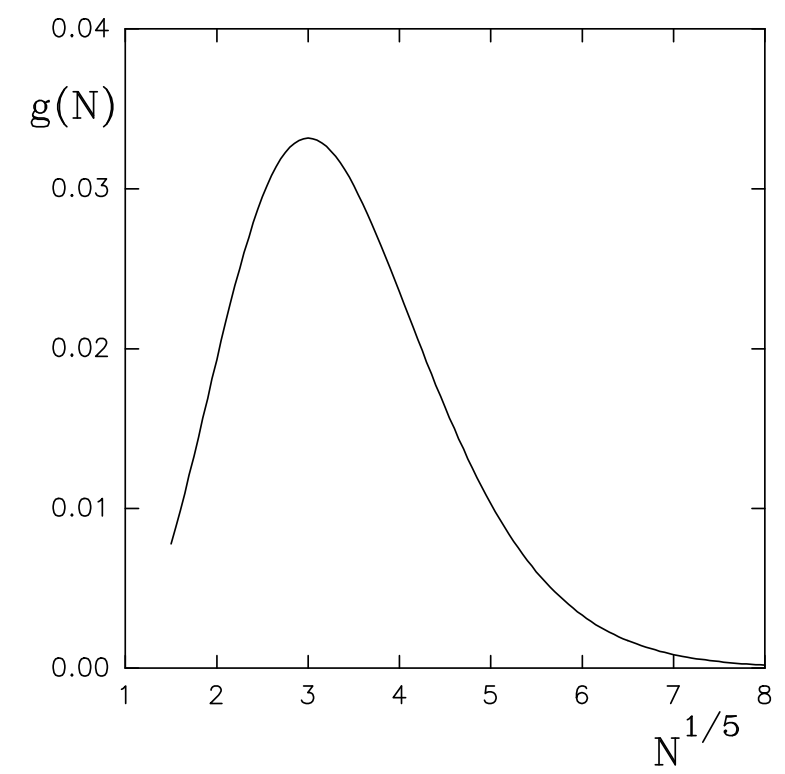

Figure 3. Dependence of fatigue function $g(N)$ by (37) on a chain length $N$. 


\title{
References
}

1. Kuhn W. // Koll. Zs., 1934, vol. 68. p. 2.

2. Volkenshtein M.V. Configurational Statistics of Polymer Chains. Moscow-Leningrad, USSR Academy of Sciences, 1959 (in Russian).

3. Flory P. Statistical Mechanics of Chain Molecules. Moscow, Mir, 1971 (in Russian).

4. Kuhn W. // Koll. Zs., 1936, vol. 76. p. 258.

5. Pietronero L. // Phys. Rev. Lett., 1985, vol. 55, No. 19, p. 2025.

6. Peliti L. Fractals Physics. - In: Proc. of the sixth Intern. Symp. on Fractals in Physics. ICTP, Trieste, Italy, July 9-12, 1985.

7. de Gennes P.G. Scaling Concepts in Polymer Physics. Ithaca, Cornell Univ. Press, 1979.

8. Gould H., Tobochnik J. An Introduction to Computer Simulation Methods. Applications to Physical Systems. Part 2. Reading, Massachusetts, Addison-Wesley Publ. Co., 1988 (Russian translation: Moscow, Mir, 1990).

9. Jones R.A.L., Norton L.J., Scull K.R. // Macromolecules, 1992, vol. 25, No. 9, p. 2359. 10. Fedoryuk M.V. Saddle-point Technique. Moscow, Nauka, 1977 (in Russian).

\section{Статистика лінійних полімерних ланцюгів у моделі випадкових блукань із самоуниканням}

\author{
Ю.Г.Медведевських
}

Відділення Інституту фізичної хімії ім. Л.В.Писаржевського НАН України 79053 Львів, вул. Наукова, За

Отримано 20 грудня 2000 р.

Запропонована точна статистика випадкових блукань із самоуниканням полімерного ланцюга у $d$-вимірному дискретному (гратка) і в неперервному просторах. Одержані асимтотичні аналітичні вирази для розподілу і густини розподілу відповідних випадкових величин, що характеризують конформаційний стан полімерного ланцюга, дана їх кількісна оцінка. Показано, що конформація полімерного ланцюга має структуру сферичної або, у більш загальному випадку, еліпсоїдної оболонки, що дифузно розмита як назовні, так і всередину полімерного клубка, ядро якого з радіусом порядка половини радіуса Флорі статистично пусте. Статистика випадкових блукань без перетинів повністю описує ефект вилученого об'єму і збігається з результатами методу Флорі у концепції П'єтронеро.

Ключові слова: метод Флорі, статистика, випадкові блукання, полімерний ланцюг, конформація, функція виживання

PACS: 05.40.Fb 